\title{
Risk Factor, Age and Sex Differences in Chronic Kidney Disease Prevalence in a Diabetic Cohort: The Pathways Study
}

\author{
Margaret K. Yu ${ }^{a, b, d}$ Courtney Rees Lyles ${ }^{c}$ Luis A. Bent-Shaw ${ }^{a, d}$ \\ Bessie A. Young ${ }^{\text {a-e }}$ and the Pathways Authors \\ ${ }^{a}$ Division of Nephrology and Departments of ${ }^{b}$ Epidemiology and ${ }^{c}$ Health Services, University of Washington, \\ ${ }^{d}$ Kidney Research Institute, and eEpidemiology Research and Information Center, Veterans Affairs Puget \\ Sound Health Care System, Seattle, Wash., USA
}

\section{Key Words}

Sex difference $\cdot$ Gender difference $\cdot$ Diabetic kidney disease

\begin{abstract}
Background/Aims: Women with diabetes experience a disproportionately greater burden of diabetic kidney disease (DKD) risk factors compared to men; however, sex-specific differences in DKD are not well defined. The effect of age on sex differences in DKD is unknown. Methods: We performed a cross-sectional analysis of the prevalence of DKD (eGFR $<60$ $\mathrm{ml} / \mathrm{min} / 1.73 \mathrm{~m}^{2}$ or microalbuminuria), advanced DKD (eGFR $<30 \mathrm{ml} / \mathrm{min} / 1.73 \mathrm{~m}^{2}$ ), and common DKD risk factors in the Pathways Study ( $n=4,839)$, a prospective cohort study of diabetic patients from a managed care setting. Subjects were stratified by age $<60$ and $\geq 60$ years to examine for differences by age. Logistic regression models examined the association between sex and prevalence of DKD and risk factors. Results: Women of all ages had 28\% decreased odds of DKD (OR $0.72,95 \% \mathrm{Cl} 0.62-0.83$ ); however, they had a greater prevalence of advanced DKD (OR 1.67, 95\% Cl 1.05-2.64), dyslipidemia (OR 1.42, 95\% Cl 1.16-1.74), and obesity (OR 1.87, 95\% Cl $1.60-2.20)$ compared to men. Women had similar odds of hy-
\end{abstract}

pertension and poor glycemic control as men. Women $\geq 60$ years had increased odds of advanced DKD, hypertension, dyslipidemia, and obesity compared to similarly aged men. Women $<60$ years had increased odds of obesity compared to their male counterparts. Conclusion: Women with diabetes had an increased prevalence of advanced DKD and common DKD risk factors compared to men and these disparities were most prominent amongst the elderly.

Copyright $\odot 2012$ S. Karger AG, Basel

\section{Introduction}

Diabetes mellitus is the leading cause of kidney failure in the United States and is associated with substantial morbidity, mortality, and health care [1]. The lifetime risk of diabetes is greater in women than in men [2] and women with diabetes have a greater prevalence of diabetic kidney disease (DKD) risk factors including hypertension [3, 4], hyperglycemia [4, 5], dyslipidemia [3-7], and obesity [4]; however, whether women have a greater prevalence of DKD is unclear. Age appears to modify the association between sex and nondiabetic kidney disease. In a large meta-analysis of nondiabetic kidney disease, Neugarten

\section{KARGER}

Fax +4161306 1234

E-Mail karger@karger.ch

www.karger.com (c) 2012 S. Karger AG, Basel

0250-8095/12/0363-0245\$38.00/0

Accessible online at:

www.karger.com/ajn
Bessie A. Young, MD, MPH

Division of Nephrology, University of Washington

1660 S. Columbian Way RDU $111 \mathrm{~A}$

Seattle, WA 98108 (USA)

Tel. +1 206277 3586, E-Mail youngb@u.washington.edu 
et al. [8] found that men had more rapid renal decline compared to women; however, a subsequent study of older subjects of postmenopausal age found that women had a faster progression of renal disease compared to men [9]. Whether there are analogous differences by age in DKD remains unknown.

Female sex has been associated with a higher risk of retinopathy and neuropathy in type 1 diabetes [10]. The effect of sex on nephropathy is not well established; however, estrogen has several effects on the kidney that may be renoprotective [11]. Estrogen deficiency accelerates the progression of glomerulosclerosis in ovariectomized mice [12] and conversely, estrogen supplementation in postmenopausal women has been shown to reduce albuminuria [13]. Furthermore, fibroblast growth factor-23 (FGF-23) levels, which predict progression to chronic kidney disease, are higher in postmenopausal women who are not on hormone replacement compared to postmenopausal women on hormone replacement or men [14]. Women with diabetes have lower levels of estradiol compared to nondiabetic women [15] and postmenopausal women with diabetes may represent a high-risk group for DKD.

In the current study, we examined the association of sex and age with the prevalence of DKD and DKD risk factors in a large cohort of outpatient diabetic patients from a vertically integrated managed care setting. We hypothesized that DKD would be more prevalent in women with diabetes compared to men and that these sex differences in DKD would be most prominent in elderly women. Additionally, we hypothesized that women with diabetes would have a higher frequency of DKD risk factors, such as hypertension, dyslipidemia, and obesity, compared to men with diabetes.

\section{Methods}

\section{Study Population}

We performed a cross-sectional analysis of baseline data from the Pathways Study, which has been described previously [16, 17]. In brief, the Pathways Study is a prospective epidemiologic survey of the prevalence and impact of depression on patients with diabetes at Group Health $(\mathrm{GH})$, a large nonprofit health maintenance organization in Washington and Idaho, USA, currently with over 500,000 enrollees. The study protocol was reviewed and approved by the institutional review boards at $\mathrm{GH}$ and the University of Washington. Nine primary care clinics were chosen for patient recruitment based on clinic size, racial/ethnic diversity, and proximity to Seattle, Wash. From these selected clinics, 9,064 potential candidates for the study were identified from the GH diabetes registry. Between 2001 and 2002, surveys were sent to these pa- tients regarding demographic information, diabetes history, and diabetes complications (retinopathy, nephropathy, neuropathy, cerebrovascular, cardiovascular, peripheral vascular disease, or metabolic disease). Of those identified, 1,222 patients were excluded from the study for the following reasons: no diabetes, gestational diabetes, cognitive impairment, severe illness, deceased, disenrollment from $\mathrm{GH}$, or language/hearing problems. Of the remaining 7,842 patients eligible for the study, 4,839 (61.7\%) returned the survey of which 4,467 (92.3\%) gave permission to link survey data with automated data from GH. Patients with a history of end-stage renal disease (ESRD; $n=67$ ) were excluded from these analyses, leaving a total of 4,400 subjects for this study.

\section{Measurements}

Automated data from GH provided information regarding laboratory tests, hospitalizations, and outpatient visits up to 18 months prior as well as pharmacy records regarding medication use up to 12 months prior to study enrollment. The average creatinine or albuminuria result was used if subjects had multiple measurements within this time frame. Estimated glomerular filtration rate (eGFR) was calculated using the Chronic Kidney Disease Epidemiology Collaboration (CKD-EPI) equations [18]. Microalbuminuria was defined as urine albumin to creatinine ratio $\geq 17 \mathrm{mg} / \mathrm{g}$ for men and $\geq 25 \mathrm{mg} / \mathrm{g}$ for women, using previously defined sex-specific cutoffs [19]. Patients with hypertension were identified by International Classification of Diseases, Ninth Revision, diagnosis codes [20]. Body mass index (BMI) was calculated from self-reported height and weight $\left(\mathrm{kg} / \mathrm{m}^{2}\right)$.

\section{Outcomes}

The primary outcomes were sex differences in the prevalence of DKD or advanced DKD at study enrollment amongst the subset of subjects with serum creatinine testing in the 18 months prior to study enrollment $(n=3,024)$. We defined DKD as the presence of either an eGFR $<60 \mathrm{ml} / \mathrm{min} / 1.73 \mathrm{~m}^{2}$ or microalbuminuria. Since a moderate decline in eGFR is normal in the elderly, we also looked at sex differences in the prevalence of advanced DKD as defined by an eGFR $<30 \mathrm{ml} / \mathrm{min} / 1.73 \mathrm{~m}^{2}$. Secondary outcomes were sex differences in the baseline prevalence of the following DKD risk factors: (1) hypertension $(\mathrm{n}=4,400)$; (2) poor glycemic control (hemoglobin A1c $\geq 8.5 \%, \mathrm{n}=4,140$ ); (3) dyslipidemia (low-density lipoprotein (LDL) $>130 \mathrm{mg} / \mathrm{dl}, \mathrm{n}=3,043$ ), and (4) obesity (BMI $\geq 30, \mathrm{n}=4,690$ ). The number of subjects in each analysis differs due to missing laboratory data.

\section{Statistical Analyses}

Statistical analyses were performed using Stata version 12 (StataCorp, College Station, Tex., USA). Significant sex differences in primary and secondary outcomes were determined using independent $t$ tests allowing for unequal variances and $\chi^{2}$ tests. Logistic regression models were used to determine if there were associations between sex and DKD prevalence and risk factors. Given the high frequency of missing LDL values (30.8\%), imputed LDL (using age, sex, race, education, chronic kidney disease (CKD) stage, hypertension, hemoglobin A1c, and BMI) was used only for the purposes of adjustment in regression models. A priori, all models were adjusted for age, race/ethnicity, education, and smoking. Models for DKD were additionally adjusted for hemoglobin Alc, imputed LDL, and BMI; hypertension was not included as a covariate because of its collinearity with the outcome. Models for
Yu/Lyles/Bent-Shaw/Young/ and the Pathways Authors 
Table 1. Pathways cohort $(n=4,400)$ patient characteristics by sex

\begin{tabular}{|c|c|c|c|}
\hline Characteristic & $\begin{array}{l}\text { Women } \\
(\mathrm{n}=2,154)\end{array}$ & $\begin{array}{l}\text { Men } \\
(n=2,246)\end{array}$ & $\mathrm{p}$ \\
\hline Age, years & $62.6 \pm 14$ & $64.0 \pm 12.6$ & $<0.001$ \\
\hline$\geq 60$ years & $1,245(57.8)$ & $1,419(63.2)$ & $<0.001$ \\
\hline Race & & & 0.45 \\
\hline Non-Hispanic white & $1,642(76.2)$ & $1,733(77.2)$ & \\
\hline Non-Hispanic black & $183(8.5)$ & $165(7.4)$ & \\
\hline Asian & $199(9.2)$ & $200(8.9$ & \\
\hline Other & $130(6.0)$ & $148(6.6)$ & \\
\hline Married & $1,130(53.0)$ & $1,750(78.3)$ & $<0.001$ \\
\hline >High school education & $1,525(71.9)$ & $1,758(79.1)$ & $<0.001$ \\
\hline Income USD $\geq 20,000 /$ year & $872(52.2)$ & $1,174(62.2)$ & $<0.001$ \\
\hline Smoker & $170(8.1)$ & $207(9.3)$ & 0.14 \\
\hline \multicolumn{4}{|l|}{ DM history } \\
\hline Type 1 DM & $242(11.3)$ & $270(12.0)$ & 0.44 \\
\hline Duration of DM, years & $9.3 \pm 9.0$ & $9.5 \pm 9.3$ & 0.50 \\
\hline Number of DM complications & $1.2 \pm 1.2$ & $1.4 \pm 1.3$ & $<0.001$ \\
\hline Hemoglobin A1c, $\%$ & $7.8 \pm 1.5$ & $7.8 \pm 1.6$ & 0.44 \\
\hline Hemoglobin A1c $\geq 8.5 \%$ & $510(25.2)$ & $522(24.7)$ & 0.67 \\
\hline Insulin & $658(30.6)$ & $648(28.9)$ & 0.22 \\
\hline Oral diabetic medications & $1,251(58.1)$ & $1,378(61.4)$ & 0.03 \\
\hline \multicolumn{4}{|l|}{ Renal function } \\
\hline Creatinine, $\mathrm{mg} / \mathrm{dl}$ & $0.9 \pm 0.4$ & $1.2 \pm 0.4$ & $<0.001$ \\
\hline $\mathrm{eGFR}, \mathrm{ml} / \mathrm{min} / 1.73 \mathrm{~m}^{2}$ & $74.4 \pm 23.3$ & $71.5 \pm 20.4$ & $<0.001$ \\
\hline Microalbuminuria & $496(33.9)$ & $686(46.5$ & $<0.001$ \\
\hline $\mathrm{DKD}, \mathrm{eGFR}<60 \mathrm{ml} / \mathrm{min} / 1.73 \mathrm{~m}^{2}$ or microalbuminuria & $799(49.6)$ & $992(56.9)$ & $<0.001$ \\
\hline Advanced DKD, eGFR $<30 \mathrm{ml} / \mathrm{min} / 1.73 \mathrm{~m}^{2}$ & $53(3.6)$ & $36(2.3)$ & 0.04 \\
\hline Hypertension & $959(44.5)$ & $904(40.3)$ & 0.004 \\
\hline \multicolumn{4}{|l|}{ Dyslipidemia } \\
\hline $\mathrm{LDL}, \mathrm{mg} / \mathrm{dl}$ & $116.0 \pm 36.1$ & $108.1 \pm 33.4$ & $<0.001$ \\
\hline $\mathrm{LDL}>130 \mathrm{mg} / \mathrm{dl}$ & $449(32.1)$ & $386(23.5)$ & $<0.001$ \\
\hline \multicolumn{4}{|l|}{ Obesity } \\
\hline BMI & $32.4 \pm 8.2$ & $29.7 \pm 5.7$ & $<0.001$ \\
\hline $\mathrm{BMI} \geq 30$ & $1,192(56.6)$ & $932(41.9)$ & $<0.001$ \\
\hline
\end{tabular}

Data are means \pm SD or numbers with percentages in parentheses. $\mathrm{DM}=$ Diabetes mellitus.

DKD risk factors were additionally adjusted for CKD stage and each of the other DKD risk factors. An interaction term between sex and age was examined in exploratory analyses of unstratified data and found to be significant $(\mathrm{p}<0.05)$ in the models for hypertension and dyslipidemia; therefore, these effects are only presented in age-stratified models. To further examine differences by age, stratified analyses by age $<60$ and $\geq 60$ years were conducted. Age 60 was used as a cutoff since prior studies in the literature found that $>99 \%$ of women were post-menopausal by the age of 57 [14]. Age-stratified analyses were adjusted for the same covariates as the unstratified analyses, with the exception of age, which was felt to be an overadjustment, as addition of age into these models resulted in similar point estimates but wider confidence intervals. All analyses were also repeated in the subset of patients with type 2 diabetes, which resulted in similar results as in the entire cohort; therefore, results are presented combined for all outcomes.

\section{Results}

\section{Pathways Cohort Characteristics}

Of the 4,400 subjects in the final cohort, $49.0 \%$ were women (table 1). Men tended to be older, married, and had higher levels of education and income than women. Among enrollees, racial composition was similar between sexes and was comprised of approximately $23.3 \%$ ethnic minorities. The prevalence of smoking was similar by sex. Overall, $11.6 \%$ of participants had type 1 diabetes. Mean duration of diabetes, hemoglobin Alc, and insulin use did not differ between men and women. Men had a higher mean number of diabetic complications compared to women $(1.4$ vs. $1.2, \mathrm{p}<0.001)$. 
Table 2. Unadjusted and adjusted odds of DKD prevalence and risk factors for women compared to men

\begin{tabular}{lll}
\hline Outcome & $\begin{array}{l}\text { Unadjusted OR } \\
(95 \% \mathrm{CI})\end{array}$ & $\begin{array}{l}\text { Adjusted OR* } \\
(95 \% \mathrm{CI})\end{array}$ \\
\hline $\mathrm{DKD}\left(\mathrm{eGFR}<60 \mathrm{ml} / \mathrm{min} / 1.73 \mathrm{~m}^{2}\right.$ or microalbuminuria) & $0.74(0.65-0.85)$ & $0.72(0.62-0.83)$ \\
Advanced DKD $\left(\mathrm{eGFR}<30 \mathrm{ml} / \mathrm{min} / 1.73 \mathrm{~m}^{2}\right)$ & $1.55(1.01-2.39)$ & $1.67(1.05-2.64)$ \\
Hemoglobin Alc $\geq 8.5 \%$ & $1.03(0.90-1.19)$ & $1.00(0.83-1.19)$ \\
BMI $\geq 30$ & $1.81(1.60-2.04)$ & $1.87(1.60-2.20)$ \\
\hline
\end{tabular}

* All models are adjusted for age, race/ethnicity, education, and smoking.

DKD models are additionally adjusted for hemoglobin A1c, LDL, and BMI. DKD risk factor models are additionally adjusted for chronic kidney disease stage and each of the other risk factors.

\section{Prevalence of DKD and Advanced DKD}

Compared to women, men had higher mean serum creatinine ( 1.17 vs. $0.92 \mathrm{mg} / \mathrm{dl}, \mathrm{p}<0.001)$, lower mean eGFR (71.5 vs. $\left.74.4 \mathrm{ml} / \mathrm{min} / 1.73 \mathrm{~m}^{2}, \mathrm{p}<0.001\right)$, and a greater prevalence of microalbuminuria ( 46.5 vs. $33.9 \%$, $\mathrm{p}<0001)$. DKD was more prevalent in men compared to women $(56.9$ vs. $49.6 \%, \mathrm{p}<0.001)$. However, advanced DKD was more prevalent in women compared to men (3.6 vs. $2.3 \%, p=0.04$ ). In adjusted logistic regression models, women had $28 \%$ decreased odds of DKD (OR $0.72,95 \%$ CI $0.62-0.83)$ but $67 \%$ increased odds of advanced DKD (95\% CI 1.05-2.64) compared to men (table 2).

\section{Prevalence of DKD Risk Factors}

Women had a greater prevalence of hypertension compared to men ( 44.5 vs. $40.3 \%, p=0.004)$. Glycemic control was similar between men and women; approximately $25 \%$ of participants had a hemoglobin A1c $\geq 8.5 \%$. Compared to men, women had higher mean LDL (116.0 vs. $108.1 \mathrm{mg} / \mathrm{dl}, \mathrm{p}<0.001)$ and a greater prevalence of LDL $>130 \mathrm{mg} / \mathrm{dl}$ (32.1 vs. $23.5 \%, \mathrm{p}<0.001)$. Moreover, women were more obese than men (mean BMI 32.4 vs. 29.7, $\mathrm{p}<0.001$ ). In multivariable logistic regression models for DKD risk factors, women had $87 \%$ greater odds of obesity (95\% CI 1.60-2.20) but similar odds of poor glycemic control compared to men (OR 1.00, 95\% CI 0.831.19).

\section{DKD and Risk Factor Prevalence Stratified by \\ Sex and Age}

Men had a greater prevalence of DKD compared to women in both age groups (table 3 ). Women $\geq 60$ years old had a greater prevalence of advanced DKD (5.2 vs. $3.3 \%, \mathrm{p}=0.04$ ), hypertension ( 54.0 vs. $46.8 \%, \mathrm{p}<0.001$ ),
Table 3. DKD and risk factor prevalence in the Pathways cohort by age and sex

\begin{tabular}{|c|c|c|c|}
\hline Outcome & Women & Men & $\mathrm{p}$ \\
\hline \multicolumn{4}{|c|}{ DKD (eGFR $<60 \mathrm{ml} / \mathrm{min} / 1.73 \mathrm{~m}^{2}$ or microalbuminuria) } \\
\hline$<60$ years & $268(41.6)$ & $294(48.4)$ & 0.01 \\
\hline$\geq 60$ years & $531(54.9)$ & $698(61.4)$ & 0.002 \\
\hline \multicolumn{4}{|c|}{ Advanced DKD $\left(e G F R<30 \mathrm{ml} / \mathrm{min} / 1.73 \mathrm{~m}^{2}\right)$} \\
\hline$<60$ years & $6(1.0)$ & $2(0.4)$ & 0.20 \\
\hline$\geq 60$ years & $47(5.2)$ & $34(3.3)$ & 0.04 \\
\hline \multicolumn{4}{|l|}{ Hypertension } \\
\hline$<60$ years & $287(31.6)$ & $240(29.0)$ & 0.25 \\
\hline$\geq 60$ years & $672(54.0)$ & $664(46.8)$ & $<0.001$ \\
\hline \multicolumn{4}{|c|}{ Hemoglobin A1c $\geq 8.5 \%$} \\
\hline$<60$ years & $268(31.6)$ & $237(30.9)$ & 0.75 \\
\hline$\geq 60$ years & $242(20.6)$ & $285(21.1)$ & 0.76 \\
\hline \multicolumn{4}{|c|}{$\mathrm{LDL}>130 \mathrm{mg} / \mathrm{dl}$} \\
\hline$<60$ years & $201(35.8)$ & $182(30.5)$ & 0.05 \\
\hline$\geq 60$ years & $248(29.6)$ & $204(19.5)$ & $<0.001$ \\
\hline \multicolumn{4}{|l|}{$\mathrm{BMI} \geq 30$} \\
\hline$<60$ years & $613(69.0)$ & $442(53.8)$ & $<0.001$ \\
\hline$\geq 60$ years & $579(47.5)$ & $490(34.9)$ & $<0.001$ \\
\hline
\end{tabular}

Data are numbers with percentages in parentheses.

and dyslipidemia ( 29.6 vs. $19.5 \%, \mathrm{p}<0.001)$ compared to men of the same age; younger subjects had no sex differences in these factors. Women were significantly more obese than men in both age groups. Glycemic control was similar between men and women in both age groups.

In multivariable logistic regression models, women had decreased odds of DKD compared to men of the same age ( $<60$ years: OR $0.66,95 \%$ CI $0.52-0.85 ; \geq 60$ years: OR $0.75,95 \%$ CI $0.62-0.91$; fig. 1 ). Women $\geq 60$ years had $63 \%$ greater odds of advanced DKD (95\% CI 1.01-2.63), 
Fig. 1. Adjusted odds of DKD prevalence and risk factors for women compared to men, stratified by age. All models are adjusted for race/ethnicity, education, and smoking. DKD models are additionally adjusted for hemoglobin A1c, LDL, and BMI. DKD risk factor models are additionally adjusted for CKD stage and each of the other risk factors.

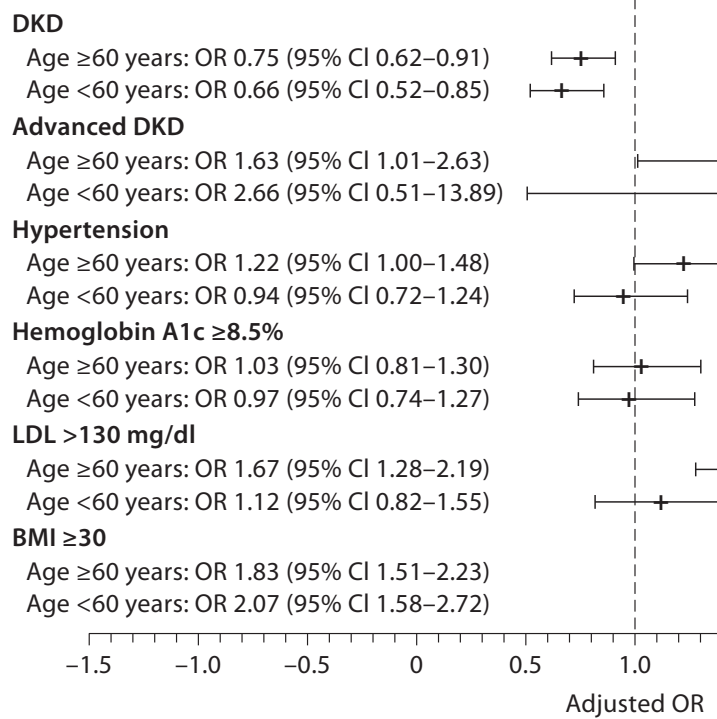

$22 \%$ greater odds of hypertension (95\% CI 1.00-1.48), and $67 \%$ greater odds of dyslipidemia (95\% CI $1.28-2.19$ ) compared to men of the same age; there were no sex-specific differences in these factors in subjects $<60$ years. Women in both age groups had approximately 2 -fold greater odds of obesity compared to their male counterparts. There were no age-stratified differences in glycemic control in the regression models.

\section{Discussion}

In this analysis of patients with diabetes from a managed care setting, we found that women had a greater prevalence of advanced DKD, hypertension, dyslipidemia, and obesity than men. These sex-specific differences were primarily accounted for by subjects $\geq 60$ years old. To our knowledge, this is the first study to report the effect of age on sex-associated differences in DKD.

While it is generally accepted that women are at reduced risk of nondiabetic kidney disease, sex differences in DKD are less clear. We found that although men with diabetes had a greater prevalence of microalbuminuria, women with diabetes were at increased odds of advanced renal dysfunction. Other studies have likewise suggested that women with diabetes have a high prevalence of impaired eGFR. In a population-based study of 3,288 patients with diabetes in the United Kingdom, women had a greater prevalence of eGFR $<60 \mathrm{ml} / \mathrm{min} / 1.73 \mathrm{~m}^{2} \mathrm{com}-$ pared to men, based on the Modification of Diet in Renal Disease (MDRD) study equation [21]. The UK Prospective Diabetes Study 74 (UKPDS) found that although men with diabetes had a higher risk of microalbuminuria, women with diabetes were at higher risk of CockcroftGault estimated creatinine clearance $<60 \mathrm{ml} / \mathrm{min} / 1.73 \mathrm{~m}^{2}$ [22]. In contrast, studies from Hong Kong [23] and Japan [24] reported a greater prevalence of DKD in men versus women, although this may be related to the racial/ethnic differences in the study populations. None of these previous studies used the CKD-EPI equations for estimating GFR, which has been shown to be more accurate than the MDRD equation in patients with diabetes [18].

Our study also found sex-related disparities in the prevalence of common DKD risk factors. Women in our study had a greater prevalence of dyslipidemia and obesity compared to men. Previous studies in academic [5], health maintenance organization [3], and populationbased [7] settings have reported that women with diabetes were more likely to have hypertension and dyslipidemia compared to men. A cross-sectional multicenter study found that women with diabetes had higher blood pressure and LDL compared to men with diabetes [25]. Although other studies reported worse glycemic control in diabetic women compared to men $[4,5,7,25]$, we did not find any sex differences in this risk factor.

We found that age affected the association between sex and DKD. Women $>60$ years of age, the majority of whom were presumably post-menopausal, had higher odds of 
advanced DKD compared to men of the same age. We are not aware of any other studies that examined sex differences in DKD by age. We also found that elderly women had greater odds of common DKD risk factors such as hypertension and dyslipidemia compared to their male counterparts, and these associations were not observed in the younger subjects. This is similar to the findings by Nilsson et al. [6] who reported that women with diabetes had worse blood pressure and glycemic control than men, and these differences were most prominent amongst patients $>60$ years of age; sex-related differences in eGFR were not examined in this study.

The mechanisms behind how age modifies DKD prevalence and risk factors may be related to hormonal changes that occur with aging. Estrogen affects the kidney by reduction of mesangial cell proliferation, increased activity of metalloproteinase enzymes and nitric oxide synthesis, changes in inflammation, decreased renin-angiotensin system activity, and decreased FGF-23 levels $[11,14]$. Other potential mechanisms for sex differences in kidney disease may be related to differential renal hemodynamics, diet, kidney/glomerular size, and sex-specific genetic polymorphisms [26].

It is important to note limitations of this study. Our study population is an insured population; therefore, generalizability of our results may be limited to those with similar access to health care. We had very few subjects $<60$ years with advanced DKD and this particular analysis was underpowered to detect sex-specific differences. Still, our study was large enough to identify sex differences in advanced DKD amongst subjects $\geq 60$ years old and to perform age-stratified analyses for other outcomes. While our study did include a small number of type 1 diabetic patients, the vast majority had type 2 diabetes, which is the largest growing population of patients developing DKD and ESRD (2). Although we did not have true GFR measurements, we used the CKD-EPI equations to estimate GFR which should be more accurate than the MDRD equation in this population.

In summary, our findings suggest that women with diabetes have an increased prevalence of advanced DKD and common DKD risk factors compared to men. These sex-related disparities were most prominent in elderly women, who represent a high-risk group for advancedstage DKD. Further studies are needed to determine whether these age and sex differences in DKD prevalence and risk factors translate into sex-specific differences in renal progression to ESRD.

\section{Acknowledgement}

This work was supported by research grants by the National Institutes of Health National Institute of Diabetes, Digestive, and Kidney Diseases (DK079745, T32 007467) and National Institute of Mental Health (MH41739, MH01643). In addition, B.A.Y.'s contribution is supported by resources from the VA Puget Sound Health Care System, Seattle, Washington.

\section{Disclosure Statement}

All authors declared no competing interests.

\section{References}

1 US Renal Data System, USRDS 2011 Annual Data Report: Atlas of Chronic Kidney Disease and End-Stage Renal Disease in the United States. National Institutes of Health, National Institute of Diabetes and Digestive and Kidney Diseases, Bethesda, MD, 2011.

2 Narayan KM, Boyle JP, Thompson TJ, Sorensen SW, Williamson DF: Lifetime risk for diabetes mellitus in the United States. JAMA 2003;290:1884-1890.

3 Ferrara A, Mangione CM, Kim C, Marrero DG, Curb D, Stevens M, et al: Sex disparities in control and treatment of modifiable cardiovascular disease risk factors among patients with diabetes: Translating Research Into Action for Diabetes (TRIAD) Study. Diabetes Care 2008;31:69-74.
4 Keane WF, Brenner BM, de Zeeuw D, Grunfeld JP, McGill J, Mitch WE, et al: The risk of developing end-stage renal disease in patients with type 2 diabetes and nephropathy: the RENAAL study. Kidney Int 2003;63: 1499-1507.

5 Wexler DJ, Grant RW, Meigs JB, Nathan DM, Cagliero E: Sex disparities in treatment of cardiac risk factors in patients with type 2 diabetes. Diabetes Care 2005;28:514-520.

6 Nilsson PM, Theobald H, Journath G, Fritz T: Gender differences in risk factor control and treatment profile in diabetes: a study in 229 Swedish primary health care centres. Scand J Prim Health Care 2004;22:27-31.

-7 Gouni-Berthold I, Berthold HK, Mantzoros CS, Bohm M, Krone W: Sex disparities in the treatment and control of cardiovascular risk factors in type 2 diabetes. Diabetes Care 2008;31:1389-1391.
-8 Neugarten J, Acharya A, Silbiger SR: Effect of gender on the progression of nondiabetic renal disease: a meta-analysis. J Am Soc Nephrol 2000;11:319-329.

-9 Jafar TH, Schmid CH, Stark PC, Toto R, Remuzzi G, Ruggenenti P, et al: The rate of progression of renal disease may not be slower in women compared with men: a patient-level meta-analysis. Nephrol Dial Transplant 2003; 18:2047-2053.

10 Monti MC, Lonsdale JT, Montomoli C, Montross R, Schlag E, Greenberg DA: Familial risk factors for microvascular complications and differential male-female risk in a large cohort of American families with type 1 diabetes. J Clin Endocrinol Metab 2007;92: 4650-4655.

11 Seliger SL, Davis C, Stehman-Breen C: Gender and the progression of renal disease. Curr Opin Nephrol Hypertens 2001;10:219-225.
Yu/Lyles/Bent-Shaw/Young/ and the Pathways Authors 
12 Doublier S, Lupia E, Catanuto P, Elliot SJ: Estrogens and progression of diabetic kidney damage. Curr Diabetes Rev 2011;7:28-34.

13 Agarwal M, Selvan V, Freedman BI, Liu Y, Wagenknecht LE: The relationship between albuminuria and hormone therapy in postmenopausal women. Am J Kidney Dis 2005 45:1019-1025.

- 14 Ix JH, Chonchol M, Laughlin GA, Shlipak MG, Whooley MA: Relation of sex and estrogen therapy to serum fibroblast growth factor 23, serum phosphorus, and urine phosphorus: the Heart and Soul Study. Am J Kidney Dis 2011;58:737-745.

$>15$ Maric C, Sullivan S: Estrogens and the diabetic kidney. Gend Med 2008;5(suppl A): S103-S113.

16 Katon W, Von Korff M, Lin E, Simon G, Ludman E, Bush T, et al: Improving primary care treatment of depression among patients with diabetes mellitus: the design of the pathways study. Gen Hosp Psychiatry 2003;25:158168.
17 Young BA, Katon WJ, Von Korff M, Simon GE, Lin EH, Ciechanowski PS, et al: Racial and ethnic differences in microalbuminuria prevalence in a diabetes population: the pathways study. J Am Soc Nephrol 2005;16: 219-228.

18 Levey AS, Stevens LA, Schmid CH, Zhang YL, Castro AF 3rd, Feldman HI, et al: A new equation to estimate glomerular filtration rate. Ann Intern Med 2009;150:604-612.

19 Mattix HJ, Hsu CY, Shaykevich S, Curhan G: Use of the albumin/creatinine ratio to detect microalbuminuria: implications of sex and race. J Am Soc Nephrol 2002;13:1034-1039.

20 Physician ICD-9-CM; in Speirs L (ed): Medicode Publications. Salt Lake City, UT, 1999.

21 Nag S, Bilous R, Kelly W, Jones S, Roper N, Connolly V: All-cause and cardiovascular mortality in diabetic subjects increases significantly with reduced estimated glomerular filtration rate (eGFR): 10 years' data from the South Tees Diabetes Mortality study. Diabet Med 2007;24:10-17.

-22 Retnakaran R, Cull CA, Thorne KI, Adler AI, Holman RR: Risk factors for renal dysfunction in type 2 diabetes: UK Prospective Diabetes Study 74. Diabetes 2006;55:18321839.
23 Luk AO, So WY, Ma RC, Kong AP, Ozaki R, $\mathrm{Ng}$ VS, et al: Metabolic syndrome predicts new onset of chronic kidney disease in 5,829 patients with type 2 diabetes: a 5-year prospective analysis of the Hong Kong Diabetes Registry. Diabetes Care 2008;31:2357-2361.

24 Ohta M, Babazono T, Uchigata Y, Iwamoto Y: Comparison of the prevalence of chronic kidney disease in Japanese patients with type 1 and type 2 diabetes. Diabet Med 2010;27: 1017-1023.

25 McFarlane SI, Castro J, Kaur J, Shin JJ, Kelling D Jr, Farag A, et al: Control of blood pressure and other cardiovascular risk factors at different practice settings: outcomes of care provided to diabetic women compared to men. J Clin Hypertens (Greenwich) 2005;7:73-80.

26 Tien KJ, Hsiao JY, Hsu SC, Liang HT, Lin SR, Chen HC, et al: Gender-dependent effect of ACE I/D and AGT M235T polymorphisms on the progression of urinary albumin excretion in Taiwanese with type 2 diabetes. Am J Nephrol 2009;29:299-308. 